\title{
5 Motivation und Engagement der Mitarbeiter
}

Hohe Kunst des Managements: Die Pflege von Motivation und Engagement. Entscheidend: Das freiwillige Moment und die Ethik der Gabe. „Ethical Moral Values“ als Fundament managerialen Denkens und Handelns. Verantwortung für den Anderen.

Gras, so sagt ein altes Sprichwort, wächst nicht schneller, wenn man daran zieht. Ein gutes Management muss sich nicht um die Motivation und das Engagement seiner Anvertrauten („Mitarbeiter") sorgen, denn diese sind in der Regel vorhanden und werden durch gutes Management ständig gefördert. Ein schlechtes Management kann jedoch beide erheblich stören, mindern oder gar zerstören. Ethische Kompetenz und Wachheit der Führungskräfte („ethical awareness") achtet die sensiblen Phänomene von Achtung, Selbstachtung und Wertschätzung und wird damit zu einem positiven Betriebsklima beitragen.

\subsection{Engagement und Patientenorientierung}

\section{Aus einem Untersuchungsbericht zur Situation eines Klinikums}

Mitarbeiter berichten häufig, dass sie nicht mehr gerne freiwillig etwas hergeben, sich nicht mehr freiwillig engagieren, weil die Leitung ihnen zu viel abpresst und sie nicht gut behandelt. Die Formel lautet: ,Wenn das Unternehmen nicht fair mit mir und anderen umgeht, dann bin ich es auch nicht. Wir strengen uns an, das Klinikum streicht die Lorbeeren ein und meckert uns an, dass es noch immer nicht genug ist. Irgendwann hörst Du auf mit Engagement. 
Wer ein Krankenhaus führt und mitgestaltet muss sich persönlich für engagierte Teams in der Patientenversorgung engagieren. Die Pflege der personellen Voraussetzungen echter Patientenorientierung aller Mitarbeiter, d.h. ihrer Menschlichkeit, ist eine Königsdisziplin für Führungskräfte. Sie sollte ein aktiv verfolgtes und kontrolliertes Ziel im Rahmen der Unternehmensstrategie sein. Nur wenn Ärzte, Therapeuten und Pflegende in jeder Hinsicht wach und aufmerksam sind, wenn sie zueinander und zu den Patienten zugewandt sind und daraus eine lebendige Verantwortung entwickeln, kann eine gute und sichere Patientenversorgung gelingen. Dafür zu sorgen ist zuallererst Aufgabe der Führungsspitze und des Top-Managements.

\section{Aufbau und Pflege der personellen Voraussetzungen echter Patienten- orientierung und Menschlichkeit der Mitarbeiter ist eine Königsdisziplin für Führungskräfte im Krankenhaus. Dies sollte ein aktiv verfolgtes und kontrolliertes Ziel der Unternehmensstrategie sein.}

So verstandenes Engagement ist nicht primär ein psychologisches Phänomen, sondern ein existenzielles und damit auch ethisches. Die Patienten, ihre Angehörigen und die Bevölkerung insgesamt erwarten von den Ärzten und anderen Heilberufen ein Engagement, das dem Ethos des Helfens und Heilens entspricht. Verantwortung ist dabei wörtlich zu verstehen als „dem Gegenüber Antwort geben“. Dazu gehört das Sehen des Patienten, das „Wahr-nehmen“, das Sprechen, das Sorgen. Die Einrichtungen des Gesundheitswesens und ganz besonders die Kliniken müssen dieses sensible und zugleich wesentliche Moment einer medizinischen Kultur pflegen und schützen. Darüber hinaus sollte auch der Politik dieses Herzstück der Gesundheitsberufe schützenswert sein, damit es nicht unter dem Zugriff von ökonomischen Kennzahlen und Kontrollparagraphen erstickt wird. Es muss sich für Krankenhäuser auch wirtschaftlich lohnen, engagierte und fähige Teams zu entwickeln.

Management muss das Engagement der Teams pflegen und erhalten. Ärzte und Pflegende, die nicht gerne ihre Arbeit machen, die erschöpft oder ,ausgebrannt“" sind oder „Dienst nach Vorschrift“ machen, können keine „gute Qualität“ der Versorgung gewährleisten. Es sind viele scheinbar kleine Einzelheiten, die über die Qualität der Versorgung entscheiden und die Sicherheit der Patienten beeinflussen. Diese entziehen sich oft der Aufsicht und der messenden Qualitätskontrolle, auch weil sie mit der richtigen Indikationsstellung und dem Auslösen medizinisch-pflegerischer Maßnahmen zusammenhängen. 
Schwestern, Pfleger und Ärzte müssen oft schnell reagieren, wenn es Patienten schlecht geht oder Anzeichen für Gefahr - beispielsweise kritische Laborbefunde oder Monitoralarme - auftauchen. Dazu braucht es Qualifikation und Wissen, Energie und Wachheit und Motivation. Gleichgültige Mitarbeiter sind im Krankenhaus fehl am Platz. Manchmal geht es um Minuten oder sogar um Sekunden, beispielweise bei einer akuten Blutung, einem Notkaiserschnitt, einem Infarkt, einem Schlaganfall oder einer psychotischen Krise. Auch in weniger dramatischen Szenen sind Aufmerksamkeit und Geduld erforderlich, um beispielsweise eine Einverständniserklärung zu bekommen oder um überhaupt das Problem des Patienten oder seiner Angehörigen zu verstehen. Selbstverständlich ist das Bemühen um die richtige Diagnose und die angemessene Therapie. Beides verlangt ein ständiges Bemühen um den Patienten und um die Erlangung des bestmöglichen Wissens.

\section{Engagements im Krankenhaus}

In der Notaufnahme eines Großklinikums herrschte seit Monaten schlechte Stimmung. Die Arbeitsbelastung war hoch, die Räumlichkeiten beengt, Angehörige von Patienten warteten ungeduldig, einige verhielten sich aggressiv. Viele Mitarbeiter waren müde, gereizt und hatten sich eine Schutzschicht von Routineverhalten umgelegt. Da wurde ein schwer verletztes Kind in lebensbedrohlichem Zustand vom Notarztwagen gebracht. Schlagartig elektrisierte sich das gesamte Team. Plötzlich liefen alle Abläufe ohne Anordnung oder Kommando. Pflegende und Ärzte arbeiteten auf Hochtouren, es wurde gerannt, gegenseitig geholfen, egal ob man den Kollegen mochte oder nicht, egal ob Arzt oder Schwester. Am Ende wurde das Kind gerettet, das Team war erschöpft, aber zufrieden. Lediglich die Fragebögen der Abteilung für Qualitätsmanagement zur Erfassung der Zufriedenheit von Patienten und Angehörigen trübte die Stimmung, denn etliche Patienten mit leichten Beschwerden, die länger warten mussten, gaben später negative Bewertungen der Abteilung zu Papier. Einige Angehörige beschwerten sich. Das Qualitätsmanagement rügte das Team, das schlechte Bewertungen erhalten hatte.

Es gehört zum Ethos der Medizin und der Heilberufe selbstlos und gerecht Hilfe zu leisten, und zwar zu jeder Zeit, Tag und Nacht, während der Arbeit, auf der Straße, selbst im Urlaub. Dieser Anspruch bleibt auch dann bestehen, wenn die Angehörigen der Gesundheitsberufe und ihre Verbände finanzielle Interessen verfolgen. Auch (und gerade!) ein um gutes Einkommen kämpfender Arzt oder Pfleger muss schnell reagieren, sich körperlich bewegen, sich in jeder Hinsicht vollständig einsetzen, wenn es die Not eines Menschen verlangt. Wirtschaftliche Ziele müssen in der Situation der Not und Gefahr eines Patienten zurückgestellt werden. Selbst, wenn einzelne Personen ihre persönlichen wirtschaftlichen Ziele 
verfolgen und auch wenn die Erwirtschaftung von Profiten das Formalziel eines privaten Klinikbetreibers ist, gehört in der konkreten Versorgungssituation die vollständige Priorität der Gesundheit und dem Leben des Patienten.

Hygienemaßnahmen, die mit über die Versorgungsqualität und die Patientensicherheit entscheiden, sind nur mit echtem Engagement der Mitarbeiter effektiv. Niemand kann hinreichend überwachen, ob die Händedesinfektion oder die Sterilitätserfordernisse beim Operationsbesteck wirklich eingehalten werden. Niemand kann kontrollieren, ob die Nachtschwester bei einem Herzstillstand sofort oder erst nach einigen Minuten das Reanimationsteam ruft. Niemand kann die Sorgfalt eines Operateurs, eines Anästhesisten oder eines Arztes im Herzkatheterlabor vollständig überwachen. Es gibt unendlich viele einzelne Tätigkeiten und Aufmerksamkeiten im Handeln des medizinischen Personals die sich nicht überwachen lassen. Solche lebenswichtigen Aufmerksamkeiten und Handlungen können nur durch die Akteure selbst erfolgen.

Es gehört zu den Aufgaben der Chefärzte und Pflegedirektoren die Qualität ihrer Teams zu pflegen. Es gehört aber letztlich auch zu den Aufgaben des gesamten Klinikmanagements, dass das Ethos der Heilberufe, unabhängig von der Person des Patienten und unabhängig von nicht-medizinischen Interessen, das Entscheiden und Handeln im Krankenhaus bestimmt. Das medizinische Ethos ist so verstanden kein Privileg einer besonderen Berufsgruppe, sondern es spiegelt die Grunderwartung der Menschen an den Arzt als Repräsentant der Medizin, an die Pflegekräfte und Therapeuten sowie an das Krankenhaus als die Institution der medizinischen Versorgung wider.

Ohne eine intrinsische Motivation kann keine hohe Versorgungsqualität erfolgen. Qualitätsmanagement und Qualitätssicherung können mit noch so ausgefeilten Kontrollen dieses Moment nicht ersetzen. Deshalb ist die Pflege dieser Motivation eine Aufgabe aller Führungskräfte, von der ärztlichen Direktion bis zum Personalmanagement und der kaufmännischen Leitung. Das Ansehen des Klinikums ist hochgradig abhängig von dem Vertrauen, das Patienten und Bürger ihm entgegenbringen können. Die vielfach beschworene „Qualität“ braucht als Fundament eine Unternehmenskultur, die das Ethos der Medizin und Heilberufe im Herzen hat.

Kennzahlen und Qualitätskontrollen versagen bei vielen für die tatsächliche Versorgungsqualität wesentlichen Details auf der unmittelbaren, schwer objektivierbaren Arbeitsebene. Hier kann medizinische Qualität nur durch die Förderung des Engagements der Mitarbeiter gesichert werden. 


\subsection{Das Moment der Freiwilligkeit}

Engagement für den Patienten bedeutet mehr als eine vertraglich geregelte Dienstleistung, die durch ein Leistungsentgelt abgeglichen wird. Das über den Vertrag hinausgehende „Plus“ ist eine freiwillige Gabe, ein Geschenk, das gerne gegeben wird, das keinen Preis kennt oder verlangt und das keine Schuldigkeit hervorruft. Es ist genau jenes Moment, das der Arbeit in der Medizin und Pflege ihren Reiz gibt und wesentlich zur Berufszufriedenheit beiträgt. Fehlt dieses Moment in der Kultur des Klinikunternehmens so kann es längerfristig zur Bedrohung führen.

Das Moment der Freiwilligkeit und Fürsorge gibt der Arbeit in der Medizin und Pflege ihren Reiz und trägt wesentlich zur Berufszufriedenheit bei. Fehlt dieses Moment in der Kultur des Klinikunternehmens hat dies ernste Folgen für den Erfolg des Krankenhauses.

In der Sprache und Denkweise eines auf Effizienz und Wettbewerb ausgerichteten Krankenhausmanagements erscheint dieses so wichtige Element guter Qualität nur selten. Vielleicht wurde das Ethos des Helfens und Heilens zu oft überhöht und missbraucht. Sicher wurde es auch als „Helfersyndrom“" psychologisiert und denunziert. Oder es wurde verwechselt mit einer realen Liebesbeziehung, die dann als Peinlichkeit und Heuchelei erlebt wird. Wie dem auch sei, das gesamte Führungsverhalten im Krankenhaus sollte die ,innere Flamme“ der Heilberufe nähren und nicht ersticken. Das ist eine Aufgabe, die nicht gegen das betriebswirtschaftliche Denken und Handeln auf der Basis von Kennzahlen und ökonomischen Parametern steht, sondern die diesem vorausgehen muss. Dabei können philosophisch ethische und auch theologische Gedanken sehr hilfreich sein.

\section{Supererogation}

Manzeschke und Brink verweisen auf einen Begriff aus der Theologie, der zwar wenig anschaulich sein mag, aber im Kern genau jenes Moment erfasst, das hier vom Management verstanden und übernommen werden sollte: „Supererogation“ bezieht sich auf ein Handeln, das sich „spontan und freiwillig (ereignet) und [...] sich durch Großherzigkeit oder Opferbereitschaft gegenüber anderen auszeichnet. Während Außenstehende die Tat als moralisch hochstehend bewundern, verstehen die Handelnden ihr Tun als selbstverständlich, als ihre Pflicht oder als das, was nötig ist. [...] Die Erzählung vom barmherzigen Samariter (Lk 10, 25-37) hat diesem Phänomen seinen Namen gegeben [...].“ 


\section{Freiwilligkeit und Unternehmenskultur}

In einem Klinikum der Grund- und Regelversorgung hatte der Vorstand eine gute Idee. Es sollte eine Patientenbibliothek eingerichtet werden. In einem Rundschreiben wurden die Mitarbeiter gebeten, Bücher zu spenden, die sie schon gelesen hatten. Die Aktion brachte keinen Erfolg. Viele Mitarbeiter hatten sich darauf geeinigt, keine Bücher zu stiften. Es wurde betont, dass man sehr gerne Bücher geben würde, dass man dies aber nicht tue, weil „die Verwaltung" jede Möglichkeit der Einsparung nutzen würde, Überstunden oft nicht bezahle, freiwilliges Engagement nicht wertschätzen würde.

In einem anderen, wirtschaftlich defizitären Krankenhaus befanden sich ein junger Assistenzarzt der Inneren Abteilung und ein kurz vor dem Ruhestand stehender chirurgischer Oberarzt nachts in der Aufnahmestation. Ein Patient erlitt einen Atemstillstand und musste reanimiert und intubiert werden. Der junge Arzt war überfordert. Die diensthabende Schwester bat den Oberarzt um Hilfe. Dieser wand sich ab mit der Bemerkung, es handele sich um einen internistischen Patienten. Er selbst sei nicht zuständig und rein formal hatte er damit Recht. Erst mit erheblicher Verzögerung leistete er Hilfe.

Es muss nicht betont werden, dass im Falle der Unterlassung medizinischer Hilfeleistung sowohl ethisch als auch rechtlich ein Verstoß gegen ärztliche Pflichten vorliegt. Wer heute in Kliniken ,vor Ort“ die Realität der Patientenversorgung beobachtet, muss eingestehen, dass auch bei vorhandenen Qualitätszertifikaten die Versorgung an „Nicht-Zuständigkeit" einzelner Personen aus Medizin und Pflege leiden kann.

Man kann diese einzelnen Personen kritisieren oder bestrafen, wird aber bei näherer Untersuchung zu dem Ergebnis kommen, dass auch ein Organisationsversagen vorliegt. Ärztliche Führungskräfte, die seit Jahren jede zweite Nacht Rufbereitschaft haben und unter permanenter Anspannung stehen, werden zum Risiko für ihre Patienten. Hier haben die Arbeitsbedingungen dazu beigetragen, die Bereitschaft schon zum ,normalen" Engagement zu vermindern.

Giovanni Maio verweist auf die Gefahr, „[...] dass wir uns durch die Übermacht ökonomischen Denkens immer mehr vom Geben selbst verabschieden. Wenn aber gerade in der Medizin nicht mehr das Geben das Bestimmende ist, dann ist es fraglich, ob wir überhaupt noch von Medizin, von einer sozialen Praxis sprechen können. "Neben der Ökonomisierung sieht Maio in der „Prozeduralisierung und Managerialisierung des Helfens“ jene Kräfte, die „[...] - so notwendig sie auch sein mögen- auch eine einseitige Blickrichtung auf die Helfersbeziehung fördern." (Maio 2014) 


\section{Das Moment der Freiwilligkeit sollte durch reflektierte Aktivität des Ma- nagements gepflegt werden.}

\subsection{Großherzigkeit als Unternehmensziel?}

Wenn Maio die „Ausrichtung am Managementdenken“ dafür mit verantwortlich macht, dass Güte, Großherzigkeit und Barmherzigkeit aus der Praxis des Helfens verdrängt werden und von einer „Marginalisierung der Grundmotivation zur Hilfe" spricht, dann spricht er zweifellos ein empirisch beobachtbares Phänomen an. Tatsächlich sind Güte und Barmherzigkeit weder in der Theorie noch in der Praxis der meisten Kliniken ernsthaft verfolgte Ziele. Wo sie, wie bei verschiedenen konfessionellen Kliniken, in den Unternehmensgrundsätzen erwähnt werden fehlen doch in der Regel für die Mitarbeiter verbindliche Übersetzungen.

Umso wichtiger ist es dann aber, genau diese Ziele in die Praxis des Managements im Sinne einer aktiven, maßnahmengestützten Förderung durch die Unternehmensführung zu integrieren. Dieses würde dann nicht nur den Patienten und Angehörigen guttun, sondern auch den Mitarbeitern aus Medizin, Pflege, Verwaltung und dem Management selbst. Es ist davon auszugehen, dass das gesamte Klinikum davon nicht nur „moralisch" sondern auch wirtschaftlich profitiert, sowohl durch die oben beschriebenen Auswirkungen auf die Qualität, als auch durch die Außenwirkung (s. Kap. 6). Manche, insbesondere die konfessionellen Krankenhausträger haben dies erkannt und versuchen, aus ethischen Zielen eine „Marke“ zu machen, welche die Besonderheit ihrer Einrichtungen charakterisiert und im politisch verordneten Wettbewerb der Krankenhäuser Vorteile bringen soll.

Der Erfolg einiger konfessioneller und frei-gemeinnütziger Krankenhäuser zeigt, dass „Ethical Moral Values“ als Fundament des Denkens und Handelns der Unternehmensführung und damit als Profil eines Krankenhauses einen unternehmerischen Wert erzeugen können.

Den konsequenten „managerialen“ Aufbau ihrer Krankenhausunternehmen auf der Basis von „Ethical Moral Values“ hatten bereits Ende des vorigen Jahrhunderts kirchliche Einrichtungen wie die evangelische Henriettenstitung Hannover oder die Kliniken der Barmherzigen Brüder in Österreich begonnen. Hintergrund war nicht die heute im Vordergrund stehende „Ökonomisierung“, sondern das Aussterben der seit dem Mittel- 
alter das west- und mitteleuropäische Hospitalwesen prägenden Schwestern- und Brüderschaften, die aus religiösen Gründen ihre Leben dem „Dienst am Kranken“ gewidmet hatten. Deren „Spirit“ sollte in eine zeitgemäße Form überführt und auf die Ebene der Organisationen übertragen werden. Hinzu kamen die Impulse der von Peter Drucker inspirierten Management-Wissenschaft, die im Sinne Druckers neben der Beachtung „harter Fakten“ auch die Notwendigkeit der Berücksichtigung „weicher Faktoren “ im Management forderte. Die nun zum Entwicklungsziel von Unternehmen erklärten Konzepte von „Corporate Identity“, „Corporate Philosophy“ und „Corporate Design“ sollten fortan die „Principles of Leadership“ beziehungsweise das Verhalten der Führungskräfte prägen.

Peter Drucker verweist in Bezug auf „harte“ und „weiche“ Faktoren auf viele Gemeinsamkeiten zwischen Management und Medizin. Beiden geht es um den praktischen Erfolg. Beide müssen harte und weiche Faktoren beachten und nutzen. Die Ausführungen Druckers haben hohe Übereinstimmungen mit denen des Arztes Victor v. Weizsäckers (s. Kap. 3):

"Management resembles medicine also, in that there is both a 'hard' side to it and a 'soft' side to it. The physician needs a lot of standard data, from taking the patient's temperature, to taking the patient's weight, and to prescribing a specific diet, or a specific medicine. But the physician also needs to look at the patient as a human being, and not as an inanimate object. Similarly, the executive in any organization, whether a business, a nonprofit-organization, or a government agency, needs both 'soft' and hard' skills." (Drucker 1997)

\title{
5.4 Aufrichtigkeit
}

\begin{abstract}
Von einer besonderen Gefahr des Missbrauchs von Ethik in Organisationen spricht der Soziologe Reimer Groenemeyer. Sie sei dann „im Begriff, eine Art Organisations-Software zu werden, die nichts anderes als die Effizienzsteigerung in der Organisation im Sinn hat. Ethik kann somit zu einem betriebswirtschaftlichen Instrument degenerieren, das zur Optimierung von Führungsstilen und Produktionsabläufen beitragen will und soll." Diese kritische Position findet man tatsächlich auch bei vielen Mitarbeitern im Krankenhaus, die sich den Vorgaben des Managements mit dem moralischen Argument versperren oder widersetzen, dass ökonomisches Denken und Handeln im Gesundheitswesen fehl am Platze sei und nicht unterstützt werden sollte.
\end{abstract}

Das positive Bekenntnis eines Krankenhausträgers zur „Ethik“ ist in Deutschland nicht unumstritten, wird aber von vielen Menschen gleichzeitig gewünscht und gefordert. In der Praxis der Ethikberatung finden 
sich immer wieder verächtliche Abwertungen „der Ethik“ durch Klinikmitarbeiter aller Ebenen und Berufsgruppen, da „Ethik“ ja nicht ernst gemeint sei und es letztendlich nur um Geld, Gewinn oder Profit gehe. „Ethik“ wird verdächtigt, ein besonders raffiniertes Instrument zu sein, Mitarbeiter mittels moralischen Drucks „über den Tisch zu ziehen“.

Die Bereitschaft und Motivation von Mitarbeitern zur Kooperation hängt tatsächlich auch von derlei moralischen Bewertungen ab. Nur wenn es überzeugend gelingt, auch das betriebswirtschaftliche Denken und Handeln ethisch zu legitimieren, indem beispielsweise „Effizienz“ nicht denunziert wird und der wirtschaftliche Erfolg eines Krankenhausunternehmens auch erkennbar dem Wohl des sozialen Gesundheitssystems und letztendlich der Gesellschaft dient, kann an eine Vereinbarkeit von „Geben und Gabe“ einerseits und „managerialem Denken“ andererseits gedacht werden. Genau diese Vereinbarkeit gilt es jedoch aktiv herzustellen. Dies kann nur in einer Atmosphäre offenen Austauschs stattfinden, in der über gemeinsame Ziele und deren Erreichung aufrichtig gesprochen werden kann.

Giovanni Maio hält eine solche Synthese offenbar für nicht möglich. Die Gabe, ihrer Natur nach stets „offen, unberechenbar und unmittelbar“, „nicht planbar, nicht berechenbar, nicht prozeduralisierbar“, entziehe sich „grundlegend dem managerialen Denken, weil man keine Regel aus der echten Gabe machen kann und weil man sie nicht universalisieren kann. Daher kann sie auch nicht in Leitlinien gegossen werden."

Wenn es zutrifft, dass das freie Geben, „offen und unmittelbar“ das ausmacht, was den Kern der medizinischen und pflegerischen Tätigkeit und Identität wesentlich bestimmt, dann muss es zu den Aufgaben der Krankenhausorganisation und ihrer Führungskräfte gehören, genau dies zu pflegen und zu schützen. Dann müsste der These von der grundlegenden Unvereinbarkeit von Management und Ethos widersprochen werden und hier eine zentrale Herausforderung für das Management gesehen werden. Dann müsste eine Synthese von Freiraum und Organisation geschaffen werden, basierend auf dem Respekt und der Wertschätzung des Identitätskerns der Heilberufe. Dann wäre der Bezug auf „Ethical Moral Values“ ernst gemeint und authentisch.

\subsection{Empathie und Verantwortung}

Es gibt viele sensible und teils wunderschöne philosophische, literarische und theologische Texte über das aus dem Inneren der menschlichen Seele kommende Geben, das trotz mancher unleugbarer Schatten doch ein Wesenszug des Bildes von der „guten Schwester“ und vom „guten Arzt“ in der Bevölkerung geblieben ist. Vieles davon wurde überhöht, pathetisch 
ausgeschmückt, zynisch belächelt oder auch hart kritisiert. Dennoch handelt es sich hierbei um ein Kernelement nicht nur im Selbstverständnis der Heilberufe, sondern auch in der therapeutischen Beziehungsarbeit. Dieses Geben ist mit starken Gefühlen besetzt. Diese sind kein melancholisches Beiwerk, sondern sie sind nach den Worten des Neurowissenschaftlers Antonio Damasio „Kronjuwelen“ der Orientierung im Zwischenmenschlichen. Führungskräfte sollten sich der Bedeutung der Gefühle der Mitarbeiter (und der eigenen) für die Qualität der Führung wie der Versorgung bewusst sein. Ein „hinein-fühlen“ in Patienten und Kollegen, die Empathie ist zugleich Voraussetzung für erfüllte Arbeit als auch Quelle einer Verausgabung, wenn es nicht gelingt, die eigenen Ansprüche an die Menschlichkeit zu erfüllen.

\section{Therapieerfolg und Beziehungsarbeit}

In einer kleinen Interview-Studie mit Physiotherapeutinnen (Bobath) wird deutlich, wie entscheidend für den Therapieerfolg eine „Beziehungsarbeit“ ist, die auf hoher Sensibilität und hohem Engagement im Sinne einer Achtsamkeit gegenüber dem Patienten beruht. Diese therapeutischen Qualitäten sind niemals vertraglich einforderbar, sie sind auch nicht beliebig wiederholbar. Sie sind aber zugleich die Grundlage echter „Qualität“. Wechselseitig gefühltes „Glück“ leitet die Therapeutin, „Gefühle“ erweisen sich im Sinne des Neurowissenschaftlers Antonio Damasio als „Kronjuwelen“ der automatisierten Steuerung von Lebensprozessen.

Gefragt danach, woran sie eine gute therapeutische Arbeit erkennen, was für sie gute „Qualität“ im therapeutischen Prozess ist, antworten die Teilnehmerinnen einer Fokus-Gruppe nicht mit Hinweisen auf Messverfahren, sondern auf Gefühle. „Wir orientieren uns an einem Gefühl und das führt uns auf eine Spur, dass wir richtigliegen.“ „Wir versuchen Glück herzustellen“. „Aufmerksamkeit und Suche nach dem Glücksmoment.“ „Wir haben viele Glücksmomente mit Eltern.“ „Wir lachen viel.“ „Da kommen oft so schöne Momente.“ „Das Kind freut sich schon beim Einbiegen in unsere Straße.“ „Das ist etwas, was das Leben auch lebenswert macht."

Die Gesprächsrunde entdeckt die Orientierung am ,Glück' als sichere ,Leitlinie‘: Glück bei Kind, Eltern und Therapeutin stellt sich beim ,Gelingen' ein, das als geglückte Bewegung zugleich Kompetenz, entdecktes Potential und ,Bindung' repräsentiert.

„Wichtig ist dieses Leise, im Moment der Anpassung, wo man merkt, das Kind fängt an etwas zu verarbeiten. Alle Beteiligten schöpfen daraus Kraft. Da entsteht auch Hoffnung, durch die Lernprozesse, die man gemeinsam gespürt hat. Da kann man auch den Eltern Hoffnung geben ..."

„Selbständigkeit fördern“, „helfen bei Erfüllung der Bedürfnisse des Lebens“, „da entsteht eine Begeisterung des Kindes, wenn es , seins' ist, was da ge- 
schieht. Mit wachsender Autonomie wächst Stolz, und das kommt zum gemeinsam empfundenen Glück hinzu."

In diesen Zitaten zeigt sich ein wesentliches Element für die Gewährleistung hoher „Qualität" in der Praxis der Gesundheitsberufe, wobei freilich Unterschiede bestehen zwischen der Arbeit von Bobath-Therapeuten, Pflegenden, Internisten, Psychiatern, Pathologen oder Chirurgen. Aber das Gemeinsame ist die „Begeisterung" oder die „Leidenschaft" in der therapeutischen, pflegerischen und gesundheitsfördernden Arbeit. Diese sind nur vordergründig psychologische Phänomene. Sie entspringen einer Bezogenheit von Menschen aufeinander, die über jedes Vertrags- und Dienstverhältnis hinausgeht.

Es steckt darin das Prinzip „Verantwortung für den Anderen“, das Emanuel Levinas formuliert hat. Verantwortung für den Anderen ist etwas, dem ich mich als Mensch gar nicht entziehen kann. Hinzu kommt das uralte hippokratische Gebot der Fürsorge und des Nicht-Schadens. Diese können stets positiv aber auch negativ berührt werden, sowohl durch die konkrete Behandlung als auch durch die Mechanismen der Organisation und durch die vorherrschende Kultur des Umganges miteinander. Und auch das Prinzip des Respektes vor der Autonomie der Menschen, sowohl Patienten als auch Kollegen hat eine große Bedeutung im Kontext von Empathie und Verantwortung.

„Das Ereignis der Ethik ist genau dieser Bruch mit der Gleichgültigkeit- wenn auch mit einer statistisch dominierten Gleichgültigkeit-, diese Möglichkeit des Einer für den Anderen.“ (Levinas 1995)

\subsection{Erfolgreiche Führungskräfte}

Der Erfolg von Führungskräften hängt in hohem Maße davon ab, ob und wie weit sie von den übrigen Mitarbeitern akzeptiert werden. Wenn diese sich „,verstanden“ fühlen wächst das Vertrauen zueinander. Compliance und Zusammenarbeit gelingen. Man spricht nicht ohne Grund vom „Stallgeruch“, der ein konstruktives Klima fördert. Dieser beruht auf Gemeinsamkeiten der Erfahrung und Kultur. Führungskräfte sollten wissen, wie ihre Mitarbeiter „ticken“, was sie motiviert und was ihnen zuwider ist. Sie sollten wissen, welchen Situationen und Konflikten Ärzte, Therapeuten und Pflegende ausgesetzt sind und wie sie damit umgehen. Sie sollten das Ethos der Heilberufe kennen, mit dem die Mehrheit von ihnen identifiziert ist, auch wenn sie von Medizin-und Pflegethik noch nichts gehört oder gelesen haben. Mit dem Ethos ist ein Stolz auf den Beruf verbunden, der keineswegs überheblich sein muss. Wird der Stolz verletzt, kommt es 
zu Demütigungen, so leiden alle - die Gedemütigten, die Demütiger, die Patienten und letztlich auch das Krankenhaus als Unternehmen. Umgekehrt können Führungskräfte ihren Mitarbeitern sehr viel abverlangen, wenn dies im Geiste echter Anerkennung und des Verstehens erfolgt. In diesem Sinne ist die emotionale Intelligenz von Führungskräften im Krankenhaus vor eine besondere Herausforderung gestellt und kann entscheidend vom professionellen Wissen um den speziellen kulturellen Geist eines Krankenhauses beziehungsweise „der Medizin“ profitieren.

\section{Ethik-Management pflegt die Motivation und das Engagement des "Health Care Teams“. Grundvoraussetzungen hierfür sind Aufrichtig- keit, Empathie und ein Verständnis für die kulturellen und ethischen Grundlagen von Medizin und Pflege.}

\section{Weiterführende Literatur}

Lohmann H, Debatin JF (Hrsg.) (2012) Neue Ärzte braucht das Land? Innovationsbaustelle Ärzteausbildung Deutschland. medhochzwei Verlag Heidelberg/Neackar

Manzeschke A, Brink A (2014) Gouvernementalität und Versprechen. Wirtschaftsphilosophische Anmerkungen zu einem Steuerungsregime am Beispiel unberechtigter Mehrforderungen in Non-Profit-Organisationen. In: Enkelmann WD, Priddat B (Hrsg.) Was ist? Wirtschaftsphilosophische Erkundungen. 297-323. Metropolis Marburg

Nink M (2014) Engagement Index: Die neuesten Daten und Erkenntnisse aus 13 Jahren Gallup-Studie. Redline Verlag München

Scholz C, Schmitt A (2011) Hochleistung braucht Dissonanz: Was Teams vom 5-Sekunden-Modell der Deutschen Kammerphilharmonie Bremen lernen können. Wiley-VCH Verlag Weinheim

\section{Zitierte Literatur}

Drucker PF (1997) Drucker on Management. Journal of East European Management Studies, Vol. 2, Nr. 1:76-92 Levinas E (1995) Zwischen uns - Versuche über das Denken an den Anderen. Hanser München/Wien Maio G (Hrsg.) (2014) Ethik der Gabe: Humane Medizin zwischen Leistungserbringung und Sorge um den Anderen, 2. Auflage. Verlag Herder Freiburg im Breisgau 